\title{
NUMERICAL AND EXPERIMENTAL STUDY OF AUXETIC CLOSED-CELL FOAMS
}

\author{
SERGE SHILKO AND DENIS KONYOK \\ Mechanics of Adaptive Materials and Biomechanics Department \\ V. Belyi Metal-Polymer Research Institute \\ National Academy of Sciences of Belarus, 246050 Gomel, Belarus
}

(Rec. 6 October 2004)

\begin{abstract}
The procedures of fabrication and testing of auxetic foams with closed cells based on foaming a liquid substance and by joining microspheres are discussed. Physically, to obtain an auxetic structure, bending rigidity of elastic rods, plates and shells should strongly depend on the initial curvature. The cells of small size are found mostly to hold their original shape. Large ones show relatively low rigidity, and would get deformed similarly to thin-walled shells when compressed with a possibility of losing stability. Thus, the volumetric compression of a foamed material is mainly realized at the expense of decreased free volume of large cells. Separation of cells according to deformation levels is found to cause auxetic elastic behavior in converted closed cells foams. Technologically, to obtain this auxetics we proposed a two-stage process. It includes the formation of concave cell structure by a permanent volumetric compression of the initial material just after foaming in the solidification state under the action of a liquid or gas. High plasticity of foam materials in this stage allow s us to obtain the re-entrant structure of cells. To obtain a material with non-convex cells we used mostly a gas or liquid under pressure as a forming instrument. After cooling the foam material shows the property of elastic (reversible) deformation. I he homogeneity and isotropy of Poisson's ratio of obtained auxetics are caused by a uniform distribution of the gas or liquid pressure on the sample surface. Some problems of Poisson's ratio minimization for foam materials we have solved by the finite element analysis.
\end{abstract}

\section{INTRODUCTION}

It's known that auxetic behavior is entirely due to the complex microstructure found in some heterogeneous materials but any intrinsic mechanical properties of the solid phase [1, 2]. In some works [3-5], starting with R. Lakes investigation [3] in 1987. the open-cell foams having a negative Poisson's ratio were fabricated. A disadvantage of the open-cell foam-based materials is their low stiffness, making them unsuitable for structural applications. The opinion that the closed-cell foams are more rigid and strong than opened-cell ones became an enticement for the study of such auxetic materials. In this paper the processing route and auxetic behavior modeling are discussed.

\section{PHYSICAL MODEL}

In contrast to the open-cell foam investigations, the fabrication, testing and modeling of closed-cell ones have been less developed [2]. The fabrication of auxetic foams is based on foaming a liquid substance or by microspheres joining. 
In the former case the closed-cell foam is formed analogous to permeable foam materials. Under usual conditions the positive Poisson's ratio values $v$ are the result of the convex shape of cell surfaces, which is conditioned, in its turn, by its energy expediency at free foaming.

Nevertheless, at definite conditions, e.g. at further volume compression, a part of the cell surface may acquire first a zeroth and then negative curvature. When the number of such inverted cells dominates, compressibility of the material rises till the appearance of the negative Poisson's ratio.

Physically, to obtain an auxetic structure, bending strength of elastic rods, plates and shells should strongly depend on the initial curvature. The cells of small size are found mostly to hold their original shape. Large ones show relatively low rigidity, and would get deformed similarly to thin-walled shells when compressed with a possibility of losing stability. Thus, the volumetric compression of a foamed material is mainly realized at the expense of decreased free volume of large cells [6. 7]. Separation of cells according to deformation levels is found to cause an auxetic elastic behavior in converted closed cells foams.

We may transfer to a mesomechanical description of a considered porous material with account of recurrence of its cells by isolating a fragment consisting of a finite number of structural elements (periodicity cells). We can thus forecast known reticular structures and purposefully design cell structures to minimize Poisson's ratio.

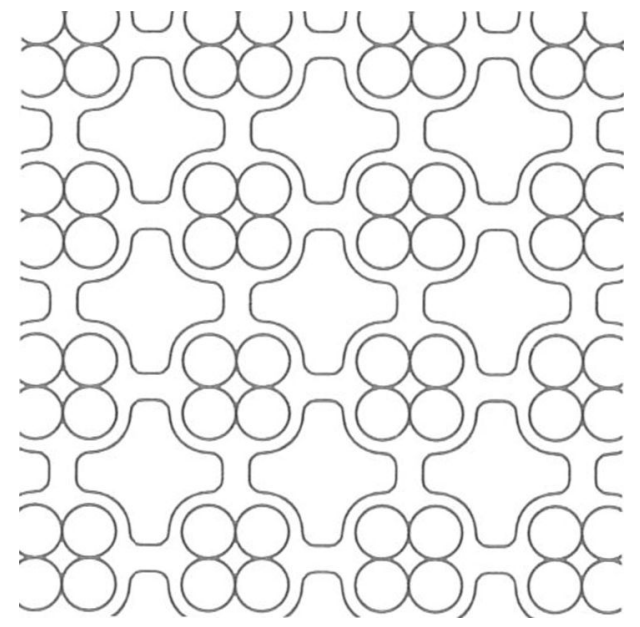

Fig. 1. Model structure of the inverted closedcell foam

Also, using the developed physical models and computer simulation it's possible to predict changes in Poisson's ratio $v$ in foams as a function of strain. So. we may study the deformation behavior of structural units consisting of a central spherical cell and symmetrically located small shells (satellites) [6]. Note please that to realize a given deformation behavior resulting in a collapse, the system of the cells should be jointly deformed symmetrically in three coordinates (Fig. 1). which avoids loss of their continuity. An expressed symmetry and sufficient number of particles may raise isotropy of the medium and its processability. 
The model of the material presented in Fig. 1 can be reached by either foaming a thermoplastic polymer material or elastomer using two fractions of a porophore or by formation of a syntact foam via regular packing and joining spherical cells of a needed size. Each structural unit of the described material presupposes transformations through the all-round compression of the initial body going simultaneously with a thermal treatment within the softening temperature range followed by cooling down to room temperature to achieve an inverted structure that preserves its isotropy of elastic properties across its main volume. The shear strength of this system depends much on deformation characteristics of the solid phase material, while its normal toughness is conditioned by susceptibility of the cells to transormations at the expense of the free volume. A final structural unit displays

- low normal stiffness in contrast to shear stiffness,

- priority transformation of the central cell due to its relatively low toughness in relation to the small-radius satellites.

\section{EXPERIMENTAL PROCEDURE}

Technologically, to obtain this auxetics we proposed a two-stage process [8. 9]. It includes the formation of a concave cell structure by a permanent volumetric compression of the initial material just after foaming in the solidification state under the action of a liquid or gas. High plasticity of foam materials in this stage allows us to obtain the re-entrant structure of cells. We have produced an effective method to obtain the material with non-convex cells mostly using a gas or liquid under pressure as a forming instrument. After cooling the foam material shows the property of elastic (reversible) deformation. Homogeneity and isotropy of Poisson's ratio of obtained auxetics are the reason of the uniform distribution of the gas or liquid pressure on the sample surface.

The first stage includes formation of cell structures of two kinds (in real conditions most probably fluctuations of size and statistical inhomogeneity of cells take place). The second stage consists of the volumetric compression of the material by applying pressure immediately after pore formation when the solid phase is still viscoplastic. As experiments have shown, an important factor is elasticity of the gas phase, which hampers inversion of the closed-cell structure.

Hence, to attain a required deformation mode the total bending strength of the walls of large cells and inner pressure of the pore-forming gas should be essentially lower in contrast to that for the small-size cells. As a result, coarse concave cells are formed. At further cooling and solidification the porous material looses its plasticity and becomes susceptible to reversible deformation characterized by the elasticity modulus and Poisson's ratio found by the known formula

$$
v=-\frac{\varepsilon_{x}}{\varepsilon_{y}},
$$

where $\varepsilon_{x}$ - relative lateral deformation; $\varepsilon_{y}-$ relative longitudinal deformation. 
So far, we may exclude the necessity of using the initial samples of a regular prismatic shape. Hence, the compression may take place immediately after formation of the foam of a random shape. To compress an open-cell material, its pores are isolated to avoid permeation of the working liquid or gas. Nevertheless, the material communicates with atmosphere for free outlet of the pore-forming gas at foaming and compression of the sample. As soon as the open-cell material with concave cells is formed a pressurized gas or liquid is fed into the insulated space to alleviate detaching of the material.

A setup made as an autoclave shown in Fig. 2 has been used in the experimental study. The setup contains body 1. cover 2. source of compressed liquid or gas 3. supply adjuster 4. tube 5. pressure gauge 6. The setup also contains the initial ingredients of the porous material 7. For transformations of the open-cell material the setup is fit with an elastic shell with antistick coat 8 communicating with atmosphere.

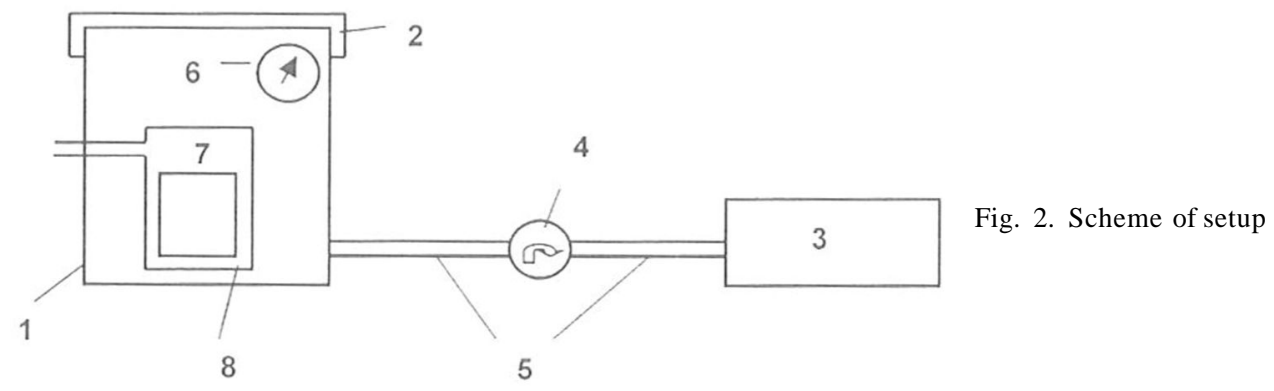

The structure of the obtained porous material has been recorded by the scanning electron microscopy using JEOL JSM-50A. The material structure at free foaming is presented in Fig. 3.

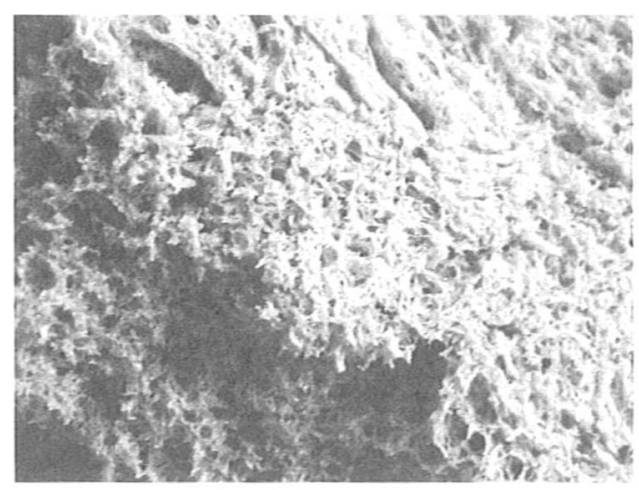

A

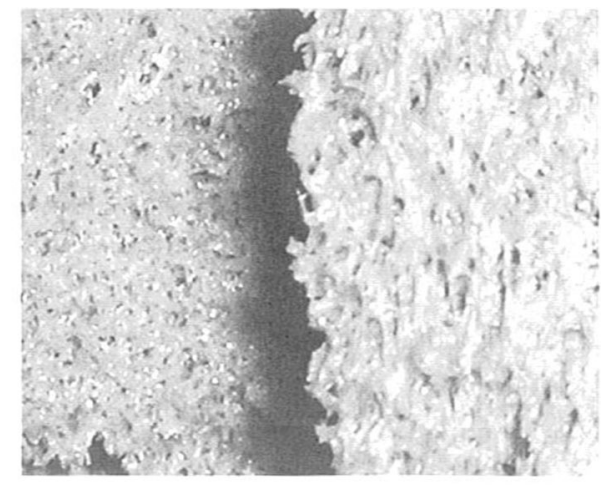

B

Fig. 3. Structure of material under free foaming (A) and under compression (B) 
Fig. 4. The individual cell of foam

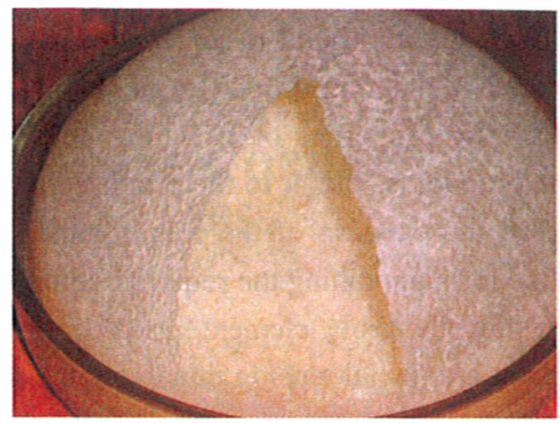

A special sample of the silicone closed-cell foam which demonstrates a 3-D fragment and a monolayer of cells shown in Fig. 4. The linear dimensions of cells have been varied within of 1-2 mm range.

\section{NUMERICAL MODELLING}

To study deformation behavior of the foam material by numerical methods we employed the finite element modeling of a fragment of two kinds of elastic porous medium with closed cells (Fig. 5).
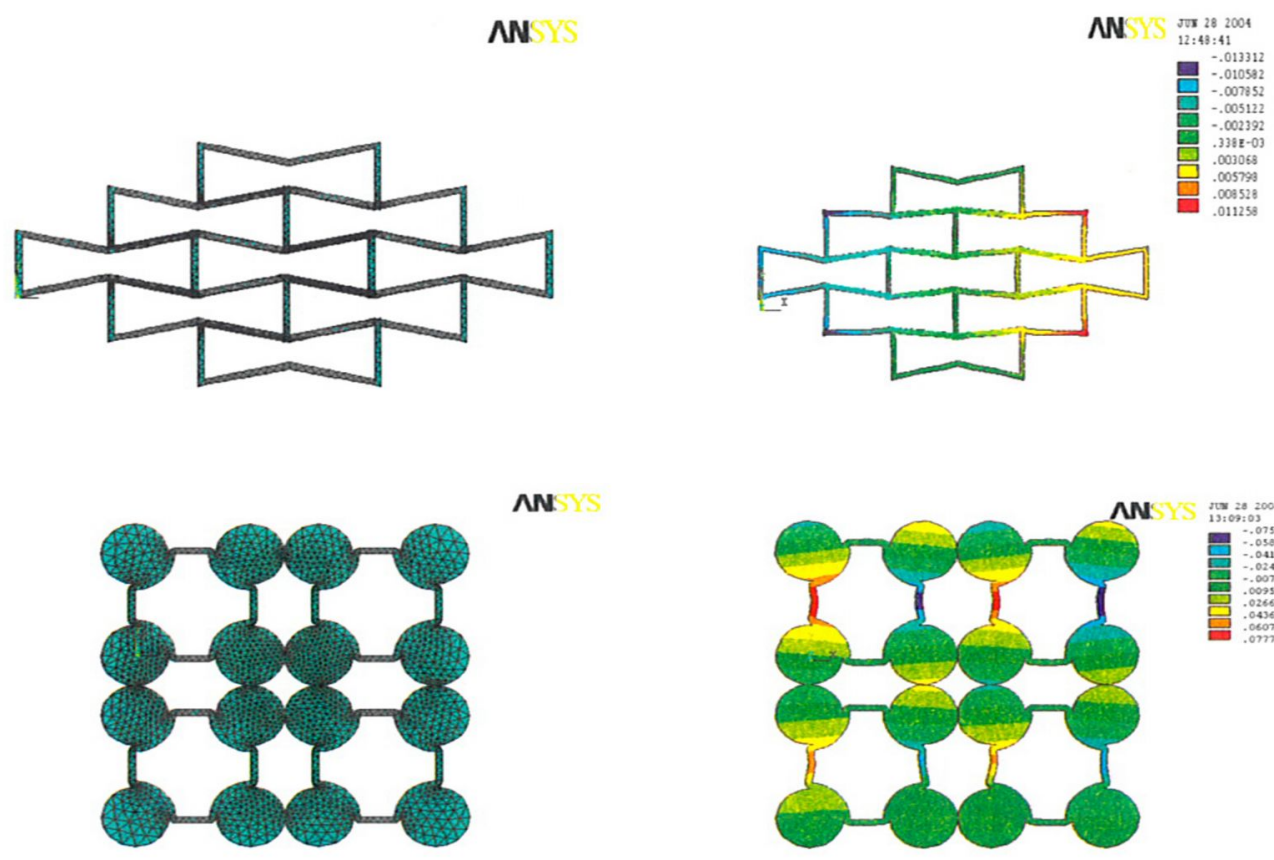

NNS

A

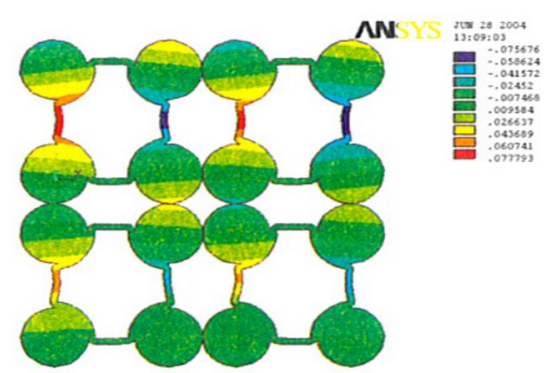

Fig. 5. Finite element models (A) and displacements calculated (B) 
Each structural unit is constructed and united with neighboring units via triangular platelet elements simulating properties of characteristic regions of the material. By varying geometry and elastic properties of structural units we can analyze various porous materials. The presented model is similar to the proposed physical one (Fig. 1) as it describes deformation of the cells at the expense of the free volume in case of the presence of the bonds between units suffice for maintaining the required deformation behavior.

Using the finite element discretization (Fig. 5b) we calculated the elastic displacement fields at a uniaxial tension due to stretching of porous material with concave cells. Each color of diagrams corresponds to equal values of transversal displacements. The calculations of fragments of two structural variants have proved that transversal (horizontal) displacements of the units belonging to the right side of the fragment are positive (Fig. 5b). This means that the both models demonstrates negative values of Poisson's ratio. Taking into account the maximal value of transversal displacements of these model structures we may obtain the estimations of Poisson's ratio: $v=-0.12$ in the first case and $v=-0.064$ in the second case (for the model with rigid round nodes).

\section{CONCLUSIONS}

A novel thermoforming processing route consisting of foaming and compression has been developed that produces an auxetic porous material with closed cells.

The finite element method may be used to predict Poisson's ratio by calculation of displacement fields of the porous structure simulated. Thus, computer analysis seems to be an effective means to design the auxetic closed-cell foams.

\section{References}

[1] K. E. Evans. Auxetic polymers: a new range of materials. Endeavour. New series (4) 170-174 (1991).

[2] D. A. Konyok. K. W. Wojciechowski. Yu. M. Pleskachevsky. and S. V. Shilko. Materials with negative Poisson's ratio (review). Mechanics of composite materials and structures. 10(1) 35-69 (2004) (in Russian).

[3] R. Lakes. Foam structure with a negative Poisson s ratio. Science. 235 1038-1040 (1987).

[4] Pat. WO 00/53830. D 01 D 5/08. Auxetic materials. Alderson K. L.. Simkins V. R. (2000).

[5] K. L. Alderson and K. E. Evans. The fabrication of microporous polyethylene having a negative Poisson's ratio. Polymer. 33(20) 4435-4438 (1992).

[6] S. V. Shilko. S. V. Stelmakh. D. A. Chernous. and Yu. M. Pleskatchevskii. Structural simulation of supercompressible materials. J. Theor. Appl. Mech. 28(1) 87-96 (1998).

[7] Y. C. Wang. R. S. Lakes, and A. Butenhoff. Influence of cell size on re-entrant transformation of negative Poisson's ratio reticulated polyurethane foams. Cell. Polym. 20. 373-385 (2001).

[8] Pat. R B 6242. B 29 C. The fabrication of material with a negative Poisson's ratio. Shilko S. V.. Konyok D. A.. Bodrunos N. N. (2002) (in Russian)

[9] Yu. M. Pleskachevsky and S. V. Shilko. Auxetic foams and composites. Poly mer Processing Simposia: Abstr. Int. Conf. Antalya. 22-24 Oct. 2001. Polymer Processing Society, Antalya. 417 (2001). 